TITLE:

\title{
Swimmers With Low Back Pain Indicate Greater Lumber Extension During Dolphin Kick and Psoas Major Tightness
}

\section{AUTHOR(S):}

Kitamura, Gakuto; Tateuchi, Hiroshige; Ichihashi, Noriaki

\section{CITATION:}

Kitamura, Gakuto ...[et al]. Swimmers With Low Back Pain Indicate Greater Lumber Extension During Dolphin Kick and Psoas Major Tightness. Journal of sport rehabilitation 2020, 29(8): 718-722

ISSUE DATE:

2020-08

URL:

http://hdl.handle.net/2433/253918

\section{RIGHT:}

Accepted author manuscriptversion reprinted, by permission, from 'Journal of sport rehabilitation", 2020, 29(6): 716-722, https://doi.org/10.1123/jsr.2018-0262.@ Human Kinetics, Inc.; この論文は出版社版でありません。引用の際 には出版社版をご確認ご利用ください。; This is not the published version. Please cite only the published version. 
1 Greater Lumbar Extension During Dolphin Kick and Psoas Major Tightness in

2 Swimmers with Low Back Pain

3

4 Authors:

5 Gakuto, Kitamura PT, B.S ${ }^{1}$, Hiroshige, Tateuchi PT, Ph.D ${ }^{1}$, Noriaki, Ichihashi

$6 \quad \mathrm{PT}, \mathrm{Ph} . \mathrm{D}^{1}$

7 1. Department of Physical Therapy, Human Health Science, Graduate School of

$8 \quad$ Medicine, Kyoto University,

9 Correspondence

10 Name: Gakuto, Kitamura, PT, B.S

11 Address: Department of Human Health Science, Graduate School of Medicine,

12 Kyoto University, 53, kawara-cho, shogoin, sakyo-ku, Kyoto 606-8507, Japan

13 Telephone: +81-075-751-3964

14 FAX: +81-075-751-3964

15 Email: kitamura.gakuto.82m@st.kyoto-u.ac.jp

16

17

18

19 


\section{Abstract}

21 Context: In competitive swimming, many swimmers experience lower back pain (LBP).

22 Lumbar hyperextension may cause LBP and tight hip flexor muscle may cause lumbar

23 extension during swimming.

24 Objective: The purpose of this study was to clarify the features of the elastic moduli of the muscles and the lumbar extension when swimmers with low back pain (LBP) perform a

26 dolphin kick (DK).

27 Design: Cross sectional study.

28 Setting: Single center.

29 Participants: Eleven male college swimmers were enrolled as the LBP group (who have LBP

30 when swimming and during a lumbar extension), and 21 male college swimmers were recruited as control group (no LBP).

Interventions: The elastic moduli of the psoas major, iliacus, teres major, latissimus dorsi,

33 pectoralis major, and pectoralis minor were measured through ultrasonic shear wave

34 elastography. The lumbar and hip extension angles during a DK were measured using a video camera. The passive hip extension and shoulder flexion range of motion (ROM) were measured using a goniometer.

Main Outcome Measures: Muscle elastic moduli and lumbar extension angles during DK. Results: The characteristics, muscle elastic moduli, DK motion, and ROM were compared between the two groups. LBP group demonstrated significantly higher elastic modulus of the psoas major and lower modulus of pectoralis minor compared to the control group. Also, LBP group showed greater lumbar extension during a DK and less hip extension ROM than the control group.

43 Conclusion: The higher elastic modulus of the psoas major and greater lumbar extension during

44 a DK may be related to the LBP in swimmers.

46 Keywords: prevention, swimming, ultrasound, posture, injury management 
In various sports, low back pain (LBP) is frequently seen in athletes including volleyball players, rhythmic gymnasts, and competitive swimming. ${ }^{1}$ This often leads to reductions in training time and/or competitive opportunities. It has been reported that about half of all competitive swimmers experience LBP. ${ }^{2}$ Therefore, the prevention of LBP in competitive swimmers is important. The physical characteristics of competitive swimmers is different from those of ordinary people. To prevent LBP in swimmers, it is necessary to know their physical characteristics. Competitive swimmers have a high risk of spinal deformities such as spine asymmetry, thoracic kyphosis, or lumbar lordosis. ${ }^{3}$ Intervertebral disc degeneration is frequently found in the lower area of the lumbar spine (mainly at the L5/S1 level, and occasionally at the L4/L5 level) and this tendency is not associated with the swimming style. ${ }^{4}$

Several studies have been conducted on the swimming kinematics of swimmers without LBP. It has been reported that the hip joint extends to about 10 degrees during a front $\mathrm{crawl}^{5}$ and may be larger during a dolphin kick (DK) owing to its performance characteristics. In a previews study, it was reported that the tightness of the hip flexor muscle can reduce hip extension that create a lumbar hyperextension and pelvic anterior tilt in various movement in water. ${ }^{6}$ Pelvic anterior tilting can make the pelvis at a lower position than normal in water. ${ }^{6}$ A study examined the swimmers experiencing LBP and reported that repetitive lumbar hyperextensions during swimming may cause LBP. ${ }^{7}$ The tight hip flexor muscle may extend lumbar spine during DK and cause LBP in swimmers. Furthermore, excessive training may also cause LBP in swimmers. However, the physical and kinematic characteristics of swimmers with LBP have not been clarified.

Competitive swimmers have more general joint laxity than normal, but the range of motion (ROM) of their internal and external shoulder rotations are smaller. ${ }^{8}$ Furthermore, it has been reported that the ROM of the internal shoulder rotations decrease as competitive swimmers continue their careers from their youth level to the college level. ${ }^{9}$ Therefore, although joint flexibility is required in competitive swimming, swimming training imposes a burden on the muscles around the shoulder joint, which can tighten. The basic posture in swimming used to achieve the least amount of water resistance, called a "streamline," is a posture with the trunk and hip at a 0 degree extension, and a shoulder flexion of greater than 180 degrees, with both hands placed above the head. As described above, swimming training can burden the muscles and tighten shoulder muscles. If a swimmer experiences tightness in the shoulder muscles, a trunk extension will occur 

as a compensation of the shoulder flexion during a streamline posture, which is frequently seen in practice. As described above, repetitive lumbar extensions can cause LBP. ${ }^{7}$ Therefore, tightness of shoulder muscles may cause wrong streamline posture that is related to LBP in swimmers.

Currently, shear wave elastography is known to be able to assess muscle stiffness noninvasively. However, no studies have compared the elastic moduli of the hip flexor and shoulder muscle or the lumbar extension angle during a DK between swimmers with and without LBP. Therefore, the aim of the present study was to compare the elastic moduli of the hip flexor and shoulder muscles as well as the ROM of the passive shoulder flexion and hip extension between swimmers with and without LBP, and to clarify the features of their muscle stiffness, hip and shoulder ROM, and lumbar motion during a DK. There were three hypotheses. First, the elastic modulus of hip flexor muscles is higher in swimmers with LBP than those without LBP. Second, the elastic modulus of shoulder muscles is higher in swimmers with LBP than those without LBP. Third, the lumbar extension angle during DK is greater in swimmers with LBP than those without LBP.

\section{METHODS}

\section{Design}

A cross sectional study was used.

\section{Participants}

Thirty-nine male college swimmers were initially enrolled in this study. The inclusion criteria of the LBP group were subjects with more than $30 \mathrm{~mm}$ of LBP in VAS while both swimming and during a lumbar extension. ${ }^{10}$ The inclusion criteria of the control group were subjects currently with no LBP $(0 \mathrm{~mm}$ on the VAS). As a result, 11 subjects were enrolled in the LBP group (21.1 \pm 1.5 years), and 21 subjects were 
enrolled in the control group (20.6 \pm 1.5 years). Three subjects were excluded because they experienced LBP when swimming but not during a lumbar extension, and four were excluded because their LBP was less than $30 \mathrm{~mm}$ in VAS. The subjects were provided a full explanation, based on the Helsinki declaration, regarding the aim, methods, and risks of the study, and their agreement was received. This study was conducted with the approval of the ethics committee of institution of authors.

\section{Procedures}

The subjects answered a questionnaire after an explanation of the study and an agreement to participate.

114 In the questionnaire, the presence and intensity of their LBP, their swimming style, and the number of their

115 training years were determined. The intensity of their LBP was assessed as being between zero and 100 based on the visual analogue scale (VAS). The elastic moduli of the psoas major, iliacus, teres major,

117 latissimus dorsi, pectoralis major, and pectoralis minor muscles were measured using ultrasonic shear wave

118 elastography. Their lumbar and hip extension angles during a DK were measured using an underwater camera. In addition, their passive hip extension and shoulder flexion ROM were measured using a goniometer.

In this study, the elastic moduli of the iliacus, psoas major were analyzed since they may restrict hip extension and the teres major, latissimus dorsi, pectoralis major clavicular, pectoralis major sternocostal, and pectoralis minor muscles were measured because they may restrict shoulder flexion. The elastic moduli of these muscles were measured using ultrasonic shear wave elastography (Aixplorer, Supersonic imagine, Aixen-Provence, France) with a linear array probe (SL10-2, Super Sonic Imagine, Aix-en-Provence, France). Using this device, the muscle stiffness can be measured quantitatively based on elastic moduli, and the elastic moduli of the muscles as measured through ultrasonic shear wave elastography were found in a study on cadavers to be strongly related to the degree of muscle expansion. ${ }^{11}$ In a living body, passive joint movement or passive tension of a muscle are strongly related to its elastic moduli. ${ }^{12}$ The elastic moduli $(G)$ reflects the elasticity or stiffness of a tissue, which is calculated based on the propagation velocity $(\mathrm{V})$ of the shear wave in the tissue, as described in the following expressions. ${ }^{13}$ 
In this expression, $\rho$ is the density of the tissue, which for muscle has been reported to be $1000 \mathrm{~kg} / \mathrm{m}^{3} .{ }^{14}$

Measurements of the elastic moduli of the psoas major and iliacus muscles were conducted in a relaxed supine position with the hip joint extended at 0 degrees, and the pelvis of the subject was fastened using a band to avoid anterior tilting. The measurement location of the psoas major and iliacus are shown in Figure 1. This location was determined to be 3 to $5 \mathrm{~cm}$ below the anterior superior iliac spine (ASIS) and distal of

142 the inguinal ligament, based on a previous study. ${ }^{15}$ The psoas major and iliacus were then identified as lateral to the femoral artery using ultrasonic images.

The shoulder position and locations of the elastic moduli measurements of the shoulder muscles are shown in Figures 2A-2C. The teres major and latissimus dorsi were measured during a relaxed sitting position and with the shoulder flexed at 90 degrees, rotated at 0 degrees, and the elbow flexed at 90 degrees. The measurement location of the teres major and latissimus dorsi was defined as $10 \mathrm{~cm}$ below the acromion

148 (Figure 2A). The pectoralis major and minor were measured during a relaxed sitting position and with the shoulder abducted at 90 degrees, rotated at 0 degrees, and the elbow flexed at 90 degrees. The pectoralis major clavicular and sternocostal parts were measured at the midpoint between the greater tubercle and the acromioclavicular joint and at midpoint between the greater tubercle and the fourth sternocostal joint, respectively. The pectoralis minor was measured at the midpoint between the coracoid process and the fourth sternocostal joint in the same way as in a previous study. ${ }^{16}$

Before measuring the elastic moduli, the state of each muscle was confirmed through ultrasonic imaging. For all muscle measurements, the region of interest (ROI), in which area the elastic moduli can be measured, was set at the center of the muscle. The elastic moduli of all muscles were measured by placing an ultrasonic probe parallel to the muscle fiber. The elastic moduli of all muscles considered were measured three times, and the mean value was used for a statistical analysis. the intra-class correlation coefficients (ICC 1.1). Consequently, the ICC 1.1 value of each muscle was as 
163 follows; psoas major $=0.888$, iliacus $=0.924$, teres major $=0.622$, latissimus dorsi $=0.756$, pectoralis major 164 clavicular $=0.755$, pectoralis major $=0.897$, and pectoralis minor $=0.642$. According to a report by Landis 165 et al., if the ICC value is higher than 0.81 , the reliability of the measurement may be nearly perfect. ${ }^{17}$ 166 Therefore, the number $(\mathrm{k})$ of measurements to meet the requirement of the lowest ICC value of the teres major, i.e., greater than 0.8, was calculated using the Spearman-Brown formula (a).

$$
\mathrm{k}=\frac{\rho 1(1-\rho 2)}{\rho 2(1-\rho 1)}
$$

In this formula, $\rho 1$ is the target ICC value, and $\rho 2$ is the resulting ICC value. As a result, all measurements of the elastic moduli were conducted three times because $\mathrm{k}$ was approximately 2.57 .

An underwater video camera (O10-SC13001-W-1, Lerving Technology, Kwai Chung N.T., Hong Kong; 1920×1080 resolution, at $60 \mathrm{fps}$ ) and a Kinovea video player (version 0.8.15, available for download at http://www.kinovea.org) were used to measure the lumbar angle during a DK. The reliability of the joint angle measurement in the sagittal plane as assessed from a digital image was obtained in a previous study. ${ }^{18}$ The subjects performed an underwater DK for $15 \mathrm{~m}$ with full effort, and the video was taken at a point 7.5 $\mathrm{m}$ away along the sagittal plane. The subjects were instructed to perform the DK at around $0.5 \mathrm{~m}$ from the surface of the water. The camera was set at $0.5 \mathrm{~m}$ below the surface of the water, $7.5 \mathrm{~m}$ from the starting side wall of the pool, and $4 \mathrm{~m}$ from the subject's swimming lane. ${ }^{19}$ Markers were put on the subject's spinous process at T10, L3, S2, iliac crest (IC), greater trochanter (GT), and lateral epicondyle of the femur (LEF) to measure the angle of the lumbar and hip joints. The lumbar angle $\left(\theta_{1}\right)$ was defined as an angle made by lines A and B. Line A was produced by the markers at T10 and L3, whereas line B was produced by the markers at L3 and S2 (Figure 3). ${ }^{20}$ The hip angle $\left(\theta_{2}\right)$ was defined as the angle made by two lines, one produced by the IC and GT, and the other produced by the GT and LEF. ${ }^{19}$ The lumbar angle was measured when the angle of the hip extension was largest when performing a DK. The average values from three appropriate trials were used in the statistical analysis and false trials in which the subjects did not pass in front of the video camera were excluded. The time for the $15 \mathrm{~m}$ DK was measured with a stopwatch. 
192 flexion ROM was assessed in a sitting position, and the hip extension ROM was assessed in a prone position.

193 All measurement of joint angle during DK and passive ROM were analyzed by the same researcher.

194

195

Statistical Analyses

196

197

198

A statistical analysis was conducted using SPSS (version 22.0, SPSS Japan, Inc., Tokyo, Japan). The

normality of all data obtained was confirmed through a Shapiro-Wilk test. The age, height, weight, number of training years, elastic modulus of each muscle, lumbar extension, and hip extension angle applied when performing a DK, as well as the passive ROM of the shoulder flexion and hip extension between the LBP and asymptomatic groups were compared using a Mann-Whitney U-test. Spearman's rank correlation coefficient test was conducted to investigate the relationship between swimming performance (best record of $50 \mathrm{~m}$ crawl or $15 \mathrm{~m}$ DK time) and degree of lumbar extension or muscle elastic modulus. The differences were shown to be statistically significant at an alpha level of 0.05 .

205

\section{RESULTS}

All measurements were completed for each subject. The characteristics of the subjects are shown in Table 1. There were no differences in age, height, weight or number of training years between both groups. The results of the elastic moduli are shown in Table 2. The LBP group showed a higher value in the elastic modulus of the psoas major compared with the control group. For the pectoralis minor, the LBP group showed a lower value compared with the control group. There were no differences in the elastic moduli of the other muscles between both groups.

The results of the lumbar and hip angle when performing a DK, along with the passive shoulder flexion and hip extension ROM, are shown in Table 3. The LBP group showed a larger lumbar extension angle when performing a DK than the control group. were no significant differences in the other joint angles between both groups. 
correlation between swimming performance and lumbar extension angle or muscle elastic modulus was

221 found (Table 4).

222

\section{DISCUSSION}

The LBP group showed a higher elastic modulus of the psoas major and lower elastic modulus of the pectoralis minor than control group. Additionally, LBP group indicated a larger lumbar extension angle when performing a DK and smaller passive hip extension angle than the control group.

This is the first study comparing the elastic moduli of the muscles and the joint angle when performing

The first hypothesis of this study was that the LBP group would show a higher elastic modulus of the hip flexor muscle than the control group. This hypothesis was partially supported because the results of this study demonstrated that, for the psoas major, the LBP group showed a higher elastic modulus than the control group, although no significant difference was shown in the elastic modulus of the iliacus. The psoas major orients from the lumbar spine, and attaches to the lesser trochanter. This muscle is a hip flexor and has an extension moment arm at levels L1 through L3 of the lumbar spine at standing and lumbar extended positions. ${ }^{21}$ Furthermore, because the spine is extended to maintain a streamline position, which is a basic position for swimming, the extension stress from the psoas major increases at a higher level of the lumbar spine. In addition, because the psoas major has a flexion moment arm at a low level of the lumbar spine, and produces a lumbar moment in a different direction between the high and low levels of the lumbar spine, it is thought that the L3 vertebra moves forward and the L1 vertebra moves backward, such that the entire lumbar spine becomes extended. ${ }^{21,} 22$ Therefore, LBP may likely occur if the elastic modulus of the psoas major is high because the lumbar spine will be more extended. Moreover, the psoas major causes a stronger shear force at the low level area of the lumbar spine than at a high level. ${ }^{21}$ It is known from studies on rats and rabbits that lumbar intervertebral disc degeneration occurs if the vertebral disc is stressed from a continual shear force. ${ }^{23,}{ }^{24}$ In humans, an overload of the intervertebral disc may be related to disc degeneration. ${ }^{25} \mathrm{~A}$ 
thickness, is related to the frequency, duration, and intensity of the LBP. ${ }^{26}$ With the subjects of our current study, an increase in the shear force at the low level of the lumbar spine may have occurred owing to the high elastic modulus of the psoas major, which could be the cause of their intervertebral disc degeneration and LBP. Although the iliacus has a hip flexion moment arm, as with psoas major, the iliacus is oriented from the pelvis and not from the lumbar spine. Therefore, if the elastic modulus of the iliacus is high, a pelvic anterior tilt and subsequent lumbar extension stress will occur; however, the stress to the lumbar spine might be less compared to that of the psoas major muscle. For this reason, it is rational for the iliacus to not be related to LBP in the present study, unlike with the psoas major.

257

The second hypothesis of this study was that the LBP group will show higher elastic moduli in the shoulder muscles than the control group; however, a lower elastic modulus was found in the pectoralis minor muscle for the LBP group than for the control group, and there were no significant differences in the elastic moduli of the other shoulder muscles. Therefore, our hypothesis was not supported. The pectoralis minor is oriented from the second to the fifth rib, and attached to the coracoid process, and the scapula is anterior tilted, rotated downward, protracted, and depressed when this muscle is activated. ${ }^{27}$ Although, the normal scapula movement during a shoulder flexion has an upward rotation, a posterior tilt, and an external rotation, ${ }^{28}$ the short length of the pectoralis minor normalized by height can cause a scapula anterior tilt and an internal rotation compared with the longer length of the pectoralis minor normalized by height. ${ }^{29}$ To the best of our knowledge, there have been no studies investigating the relationship between the stiffness of the pectoralis minor and scapula kinematics; however, if the pectoralis minor is tight, the scapula kinematics may be similar to the kinematics under a short pectoralis minor. Conversely, the scapular kinematics under less stiffness of the pectoralis minor may be similar to that of a long pectoralis minor. In this study, a low elastic modulus of the pectoralis minor in the LBP group may help the scapula tilt at the posterior and rotate externally during a shoulder flexion, and the LBP subjects may be able to compensate for a glenohumeral

273 flexion by increasing the movement of their scapula. Therefore, to prevent LBP from occurring owing to a 274 lumbar extension, the LBP group may compensate for the lumbar extension during a shoulder flexion while in the streamline position through an increased scapula movement. We initially hypothesized that the LBP group would show higher elastic moduli of the shoulder muscles than the control group because the LBP subjects would compensate for a shoulder flexion occurring from the high elastic moduli of their shoulder 
muscles by increasing their trunk extension, thereby causing LBP. However, the LBP group showed a lower

279 elastic modulus of the pectoralis minor than the control group, and accordingly, it is thought that the low elastic modulus of the pectoralis minor in the LBP group is not the cause of, but rather a compensation for, LBP. In addition to the pectoralis minor, the elastic moduli of the latissimus dorsi, teres major, and pectoralis major, as the shoulder muscles considered in this study, were measured. These muscles have a shoulder extension moment in a shoulder flexed position, ${ }^{30}$ and are thought to restrict a shoulder flexion, induce a lumbar extension, and result in LBP if their elastic moduli are high. However, there was no significant difference in the elastic moduli of the latissimus dorsi, teres major, and pectoralis major between the LBP and control groups. Therefore, it is thought that these muscles did not affect the LBP in the swimmers considered in this study.

The third hypothesis was that the LBP group would show a greater lumbar extension when performing a DK than the control group. The LBP group showed a significantly higher lumbar extension angle during a DK than the control group, which is congruent with this hypothesis. Because repetitive lumbar hyperextensions while swimming can induce LBP, ${ }^{7}$ the large angle of the lumbar extension during a DK can cause LBP. In this study, the passive hip extension ROM in the LBP group was significantly lower than that in the control group, and the subjects in the LBP group may have compensated for a hip extension when performing a DK through a lumbar extension. However, because the angle of the hip extension during a DK was about 9 degrees in both groups, it is thought that the hip was not extended to the limit of a passive hip extension ROM when performing a DK. Therefore, the significant difference in the lumbar extension angle during a DK may be influenced by another factor. The results of this study indicate that the elastic modulus of the psoas major was higher in the LBP group than in the control. Because the high elastic modulus of the psoas major can cause a lumbar lordosis, and the hip will extend the psoas major when performing a DK, thereby increasing the elastic modulus of psoas major, the high lumbar extension angle during a DK in the LBP group may be caused by the high elastic modulus of the psoas major. An approach to decrease the elastic modulus of the psoas major, or exercising the hip extension without a trunk extension may be useful in treating or preventing LBP. 
as demonstrated by the Spearman's rank correlation coefficient test. Therefore, increased lumbar extension

308 during DK does not necessarily improve the swimming performance. However, it may increase the lumbar spine load. Thus, higher lumbar extension degree during DK is not an appropriate technique to improve swimming performance or reduce lumbar spine load.

311

There may be several factors causing LBP in swimmers; extended training period being one of them.

313 However, no significant relationship was found between LBP and number of training years using Mann-

314 Whitney U-test. These results may be partially explained by the participants' team. In this study, the participants were the members of a swimming club. Thus, their practice frequency and training year were similar. So, the effects of extended training on swimmers with LBP are unclear.

There are certain limitations to the present study. First, as a cross-sectional study, it is impossible to indicate the cause and effect relationship between LBP and the elastic moduli of the muscles or the lumbar angle during a DK. Second, the inclusion criteria for the LBP in this study differ from those of many other previous studies because many other studies include LBP patients whose LBP has continued for longer than 3 months, and thus the results of this study are incommensurable with those of other studies. Third, the stiffness of the psoas major is thought to cause a lumbar extension when swimming; however, swimming styles other than a DK were not assessed. In competitive swimming, swimmers spend a significant amount of their training time using a crawl stroke regardless of their particular swimming style. Therefore, the lumbar motion during a crawl stroke may be related to LBP. To clarify the cause and effect relationship between the elastic moduli of the muscles and LBP, or the same criteria of LBP as applied in other studies, further longitudinal researches are needed.

\section{CONCLUSION} elastic modulus of their psoas major, lower elastic modulus of their pectoralis minor, and a greater lumbar extension during a DK than swimmers without LBP. This study also showed that lumbar extension during 
336 swimmers with LBP must be resolved to prevent LBP.

337

338

\section{Reference}

339 1. Vidal-conti J. The relationship between low back pain and sport practice in young people. International Journal of Orthopedics and Rehabilitation. 2014;1:67-73.

341 2. Masiero S, Carraro E, Celia A, Sarto D, Ermani M. Prevalence of nonspecific low back pain in schoolchildren aged between 13 and 15 years. Acta Paediatr. 2008;97(2):212-216.

343 3. Zaina F, Donzelli S, Lusini M, Minnella S, Negrini S. Swimming and spinal deformities: A crosssectional study. J Pediatr. 2015;166(1):163-167.

345 4. Kaneoka K, Shimizu K, Hangai M, et al. Lumbar intervertebral disk degeneration in elite competitive swimmers: a case control study. Am J Sports Med. 2007;35(8):1341-1345.

5. SANDERS RH. Kinematics, coordination, variability, and biological noise in the prone flutter kick at different levels of a "learn-to-swim” programme. J Sports Sci. 2007;25(2):213-227.

6. Kenal KA, Knapp LD. Rehabilitation of injuries in competitive swimmers. Sports Medicine.

350 1996;22(5):337-347.

351

7. Wanivenhaus F, Fox AJS, Chaudhury S, Rodeo SA. Epidemiology of injuries and prevention strategies in competitive swimmers. Sports health. 2012;4(3):246-251.

353

8. Jansson A, Saartok T, Werner S, Renström P. Evaluation of general joint laxity, shoulder laxity and mobility in competitive swimmers during growth and in normal controls. Scand J Med Sci Sports. 2005;15(3):169-176.

356

9. Riemann BL, Witt J DG. Glenohumeral joint rotation range of motion in competitive swimmers. $J$ Sports Sci. 2015;29:1191-1199.

358

10. Teodorczyk-Injeyan JA, McGregor M, Triano JJ, Injeyan HS. Elevated Production of Nociceptive CCchemokines and sE-selectin in Patients with Low Back Pain and the Effects of Spinal Manipulation. Clin J Pain. 2017;0:1.

11. Eby SF, Song P, Chen S, Chen Q, Greenleaf JF, An KN. Validation of shear wave elastography in skeletal muscle. J Biomech. 2013;46(14):2381-2387. medial gastrocnemius muscle belly using supersonic shear imaging. J Biomech. 2012;45(6):978-984. 
365 13. Hug F, Tucker K, Gennisson JL, Tanter M, Nordez A. Elastography for muscle biomechanics: Toward the estimation of individual muscle force. Exerc Sport Sci Rev. 2015;43(3):125-133.

14. Gennisson JL, Cornu C, Catheline S, Fink M, Portero P. Human muscle hardness assessment during incremental isometric contraction using transient elastography. J Biomech. 2005;38(7):1543-1550.

15. Jiroumaru T, Kurihara T, Isaka T. Establishment of a recording method for surface electromyography in the iliopsoas muscle. J Electromyogr Kinesiol. 2014;24(4):445-451.

16. Umehara J, Nakamura M, Fujita K, et al. Shoulder horizontal abduction stretching effectively increases shear elastic modulus of pectoralis minor muscle. J Shoulder Elbow Surg. 2017;26(7):1159-1165.

17. Landis JR, Koch GG. The measurement of observer agreement for categorical data. Biometrics. 1977;33(1):159-174.

18. Moral-Muñoz JA, Esteban-Moreno B, Arroyo-Morales M, Cobo MJ H-VE. Agreement between faceto-face and free software video analysis for assessing hamstring flexibility in adolescents. $J$ Strength Cond Res. 2015;29(9):2661-2665.

19. Atkison RR, Dickey JP, Dragunas A, Nolte V. Importance of sagittal kick symmetry for underwater dolphin kick performance. Hum Mov Sci. 2014;33(1):298-311.

380

20. Claus AP, Hides JA, Moseley GL, Hodges PW. Is “ideal” sitting posture real?: Measurement of spinal curves in four sitting postures. Man Ther. 2009;14(4):404-408.

382

21. Bogduk N, Pearcy M, Hadfield G. Anatomy and biomechanics of psoas major. Clin Biomech (Bristol, Avon). 1992;7(2):109-119.

22. Penning L. Psoas muscle and lumbar spine stability: A concept uniting existing controversies. Critical review and hypothesis. Eur Spine J. 2000;9(6):577-585.

23. Kim J, Yang SJ, Kim H, Kim Y, Park JB, Dubose C LT. Effect of shear force on intervertebral disc ( IVD ) degeneration : An In vivo rat study. Ann Biomed Eng. 2012;40(9):1996-2004.

24. Xia DD, Lin SL, Wang XY, Wang YL, Xu HM, Zhou F TJ. Effects of shear force on intervertebral disc : an in vivo rabbit study. Eur Spine J. 2015;24:1711-1719.

25. Stokes IAF, Iatridis JC. Mechanical conditions that accelerate intervertebral disc degeneration: Overload versus immobilization. Spine. 2004;29(23):2724-2732. pain history and lumbar MRI findings. Spine. 2003;28(6):582-588. 
394 27. Schenkman M, Rugo de Cartaya V. Kinesiology of the shoulder complex. J Orthop Sports Phys Ther. 395 1987;8(9):438-450.

396 28. McClure PW, Michener LA, Sennett BJ, Karduna AR. Direct 3-dimensional measurement of scapular 397 kinematics during dynamic movements in vivo. J Shoulder Elbow Surg. 2001;10(3):269-277.

398 29. Borstad JD, Ludewig PM. The effect of long versus short pectoralis minor resting length on scapular 399 kinematics in healthy individuals. J Orthop Sports Phys Ther. 2005;35(4):227-238.

$400 \quad 30 . \quad$ Kuechle DK, Newman SR, Itoi E, Morrey BF. Kuechle--Shoulder muscle moment arms during horizontal flexion and elevation. J Shoulder Elbow Surg. 1997;6(5):429-439.

402 


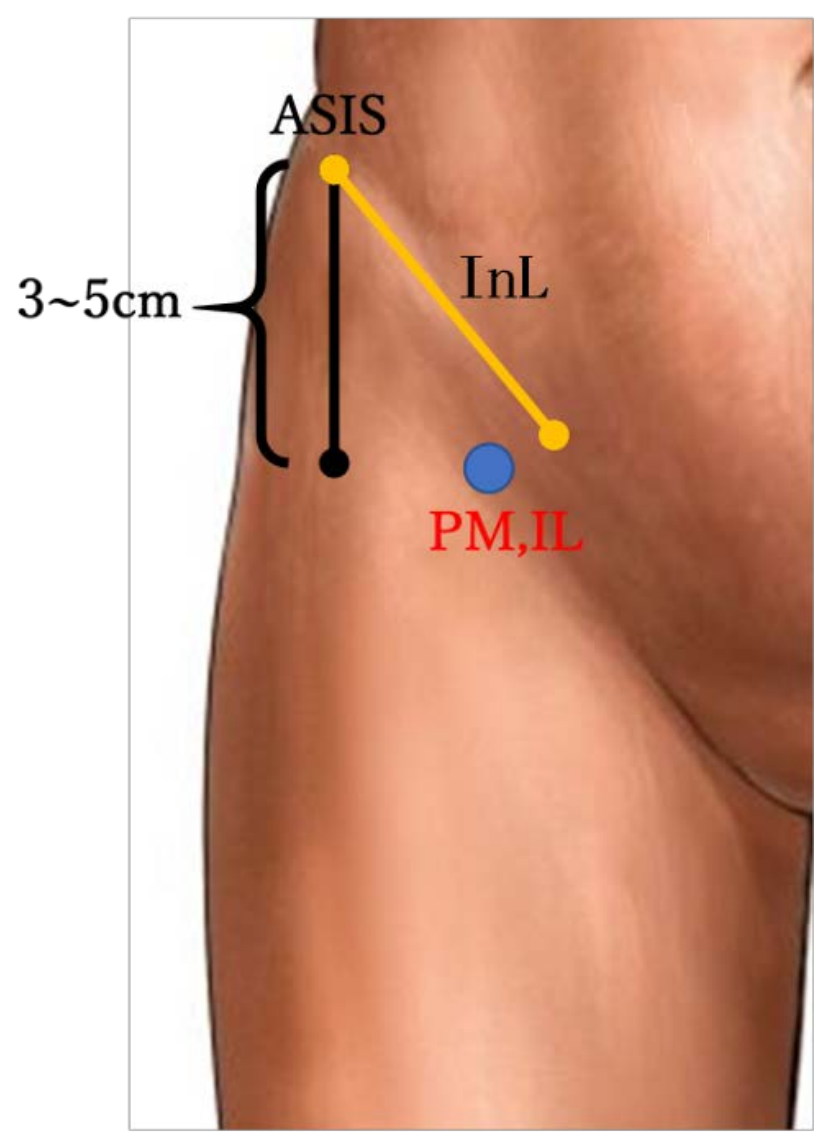

404 Figure 1. Measurement cites of shear elastic modulus for psoas major and iliacus muscles

405

406 The measurement locations of the psoas major (PM) and iliacus (IL) are shown. First, the femoral artery

407 was identified through ultrasonic imaging as 3 to $5 \mathrm{~cm}$ below the anterior superior iliac spine (ASIS) and

408 distal of the inguinal ligament (InL). These muscles were then identified lateral to the femoral artery. At

409 this location, the probe was set parallel to the muscle fiber, and the elastic moduli were measured. 


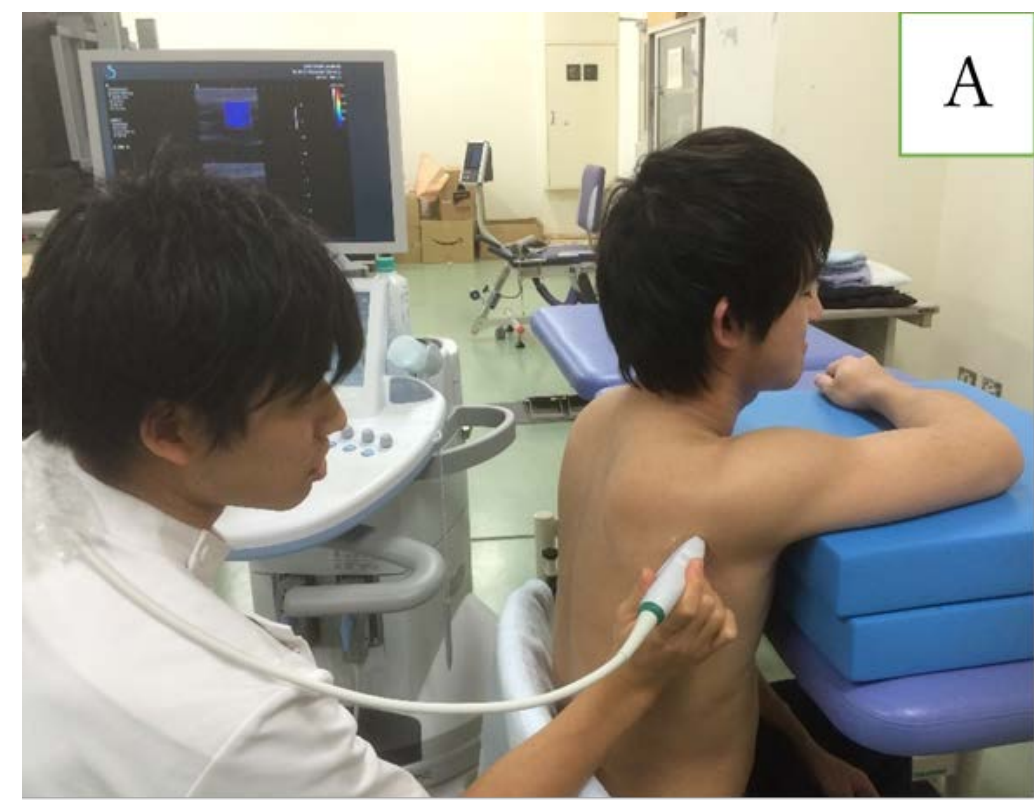

411 Figure 2A. Measurement position for the shear elastic modulus of the teres major and latissimus dorsi

413 The measurement position for the teres major and latissimus dorsi is shown in Figure 2A. The subject sat

414 with a shoulder flexed at 90 degrees, rotated at 0 degrees, and the elbow flexed at 90 degrees. 


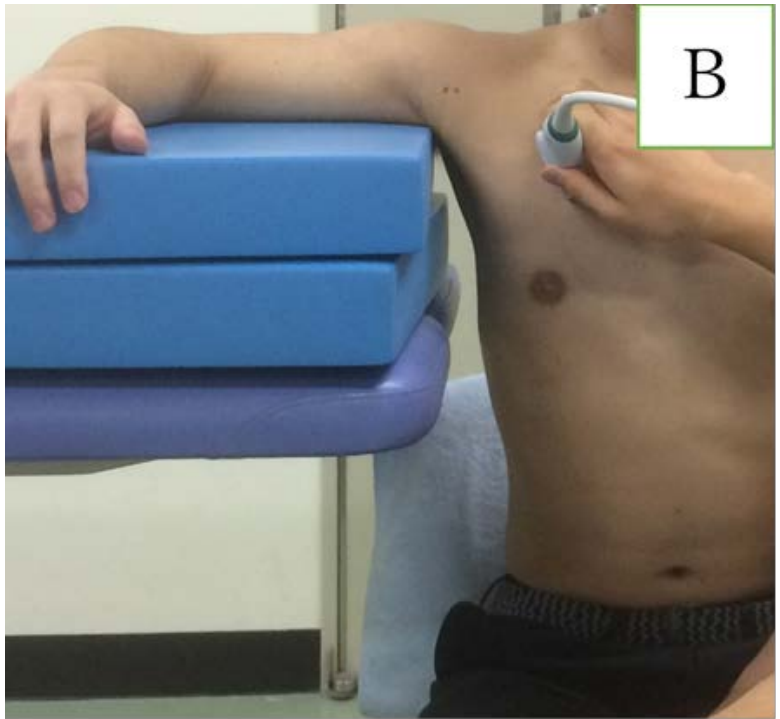

416 Figure 2B. Measurement position for the shear elastic modulus of the pectoralis major and minor shoulder

417 muscles

418

419 The position used during the measurement of the pectoralis major and minor is shown in Figure 2B. The

420 position was the shoulder abducted at 90 degrees, rotated at 0 degrees, and with the elbow flexed at 90

421 degrees.

422 


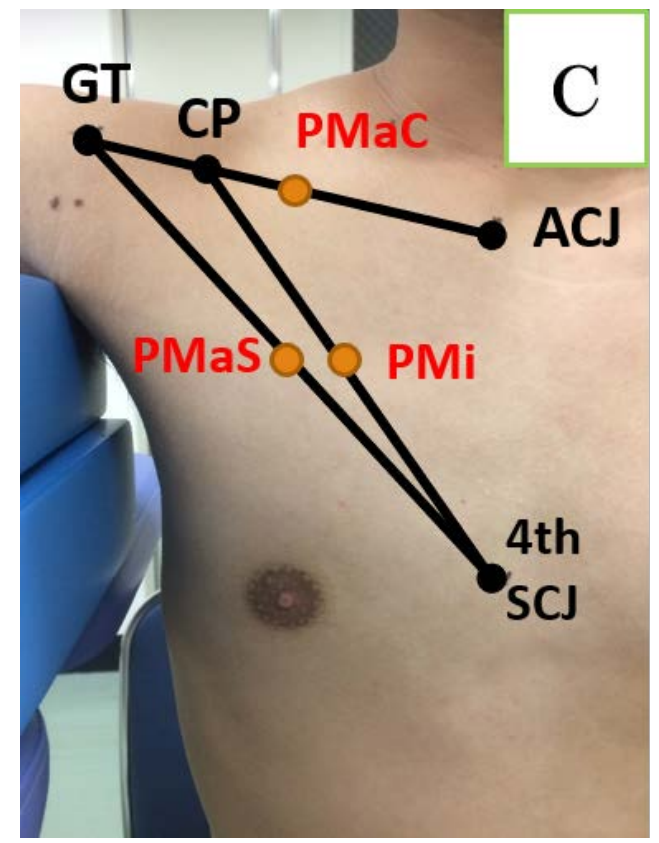

424 Figure 2C. Measurement sites of shear elastic modulus for pectoralis major clavicular part, sternocostal

425 part, and pectoralis minor muscles

426

427 The measurement locations of the pectoralis major clavicular (PMaC), sterncostal (PMaS), and pectoralis 428 minor (PMi) are shown in Figure 2C. The pectoralis major clavicular part was measured at the midpoint 429 between the greater tubercle (GT) and acromioclavicular joint (ACJ), the pectoralis minor (PMi) was 430 measured at the midpoint between the coracoid process (CP) and fourth sternocostal joint (4th SCJ), and 431 the sterncostal (PMaS) was measured at the midpoint between the greater tubercle and the fourth 432 sternocostal joint. 
433

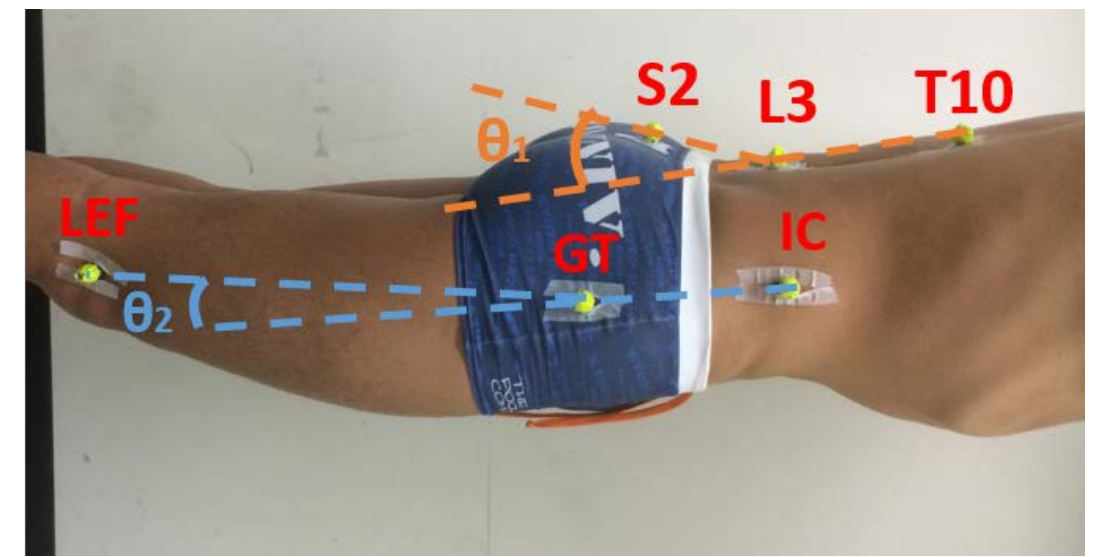

434 Figure 3. Measurement method of lumbar and hip joint angle during a DK

435

436 The measurement method of the lumbar and hip joint angle is shown in Figure 3. The lumbar angle $\left(\theta_{1}\right)$

437 was defined as the angle created by two lines, one produced by the markers at T10 and L3, and the other

438 produced by the markers at L3 and S2. The hip angle $\left(\theta_{2}\right)$ was defined as the angle made by two lines, one 439 produced by the iliac crest (IC) and greater trochanter (GT), and the other produced by the GT and lateral 440 epicondyle of the femur (LEF).

441 
442 Table 1. Characteristics of the participants

443

\begin{tabular}{lccc}
\hline & LBP group (n= 11) & Control group (n= 21) & P value \\
\hline age, y & $21.1(1.5)$ & $20.6(1.5)$ & .558 \\
height, cm & $176.4(5.7)$ & $173.2(5.9)$ & .208 \\
body mass, kg & $68.3(8.0)$ & $65.9(7.2)$ & .238 \\
training years, y & $12.6(3.5)$ & $12.1(3.4)$ & .785
\end{tabular}

444 Abbreviations: LBP, low back pain.

445

446 
447 Table 2. Shear elastic modulus of each muscle

448

Shear elastic modulus, kPa

\begin{tabular}{lcccc}
\hline Muscles & LBP group (n = 11) & Control group (n = 21) & Effect size (r) & P value \\
\hline psoas major & $17.9(4.3)$ & $13.1(2.6)$ & 0.516 & .003 \\
iliacus & $14.2(4.7)$ & $11.6(2.5)$ & 0.228 & .208 \\
teres major & $9.4(3.6)$ & $8.5(1.7)$ & 0.140 & .434 \\
latissimus dorsi & $8.8(2.6)$ & $8.1(1.6)$ & 0.088 & .639 \\
pectoralis major & $10.3(3.8)$ & $10.4(3.4)$ & 0.025 & .907 \\
clavicular part & & & & \\
pectoralis major & $8.5(3.7)$ & $8.3(2.8)$ & 0.025 & .907 \\
sterncostal part & & & & .027 \\
pectoralis minor & $5.4(2.1)$ & $7.6(3.5)$ & 0.389 & \\
\hline
\end{tabular}

449 Abbreviations: LBP, low back pain.

450 
452

\begin{tabular}{lcccc}
\hline Joint angle, deg & & & & \\
\hline & LBP group (n = 11) & Control group (n = 21) & Effect size (r) & P value \\
\hline Lumbar extension & $22.1(4.0)$ & $15.3(2.6)$ & 0.701 & $<.001$ \\
during DK & & & & \\
Hip extension & $9.5(4.3)$ & $8.7(5.3)$ & 0.070 & .690 \\
during DK & & & & \\
Passive & $199.5(11.4)$ & $202.9(8.8)$ & 0.107 & .546 \\
shoulder flexion & & & 0.364 & .040 \\
Passive & $23.6(7.4)$ & & & \\
hip extension & & & & \\
\end{tabular}

453 Abbreviations: DK, dolphin kick; LBP, low back pain. 
454 Table 4. Correlation Between Swimming Performance and Lumbar Extension or Muscle Elastic

455 Modulus

\begin{tabular}{|c|c|c|c|c|}
\hline \multirow[b]{2}{*}{ Variables } & \multirow{2}{*}{$\begin{array}{l}\text { Correlation coefficient } \\
\text { Best records of } 50 \mathrm{~m} \text { crawl }\end{array}$} & \multirow[b]{2}{*}{$P$ value } & \multirow{2}{*}{$\begin{array}{c}\text { Correlation coefficient } \\
15 \mathrm{~m} \text { DK time }\end{array}$} & \multirow[b]{2}{*}{$P$ value } \\
\hline & & & & \\
\hline Lumbar extension angle & .088 & .630 & .101 & .583 \\
\hline Psoas major & -.033 & .859 & .037 & .839 \\
\hline Iliacus & -.009 & .962 & .158 & .387 \\
\hline Teres major & .175 & .337 & .031 & .865 \\
\hline Latissimus dorsi & .219 & .229 & .229 & .207 \\
\hline Pectoralis major & -.084 & .647 & -.060 & .745 \\
\hline \multicolumn{5}{|l|}{ clavicular part } \\
\hline Pectoralis major & .041 & .826 & -.012 & .949 \\
\hline \multicolumn{5}{|l|}{ sterncostal part } \\
\hline Pectoralis minor & .129 & .483 & .063 & .734 \\
\hline
\end{tabular}

